\title{
Role of minimal hepatic encephalopathy in road traffic accidents
}

\author{
Mohammad Mohai El-Din Awad ${ }^{1 \wedge}$, Abd-El Raouf Mohamed El-Deib ${ }^{1}$, Fadia Mostafa Attia ${ }^{2}$, Mohamed Negm $^{3^{*}}$, \\ Mohamed Hassan Mohamed Soliman ${ }^{1}$ and Wafaa Hassan Omar ${ }^{4}$
}

\begin{abstract}
Background: The term minimal hepatic encephalopathy (MHE) is defined as encephalopathy that does not lead to clinically overt cognitive dysfunction but can have an impact on quality of life, risk of involvement in road traffic accidents, and ability to function in daily life.

Objective: To identify the incidence of minimal hepatic encephalopathy in patients actively involved in a road traffic accident and its role in the increased incidence of road traffic accidents.

Patients and methods: We included 74 patients presented in road traffic accidents (drivers, history of fall, pedestrians whom accidents occur during crossing the road) with clinically proven hepatic cirrhosis, without clinical manifestation of hepatic encephalopathy (HE). Thorough history taking, clinical examinations, routine laboratory investigations, serum IL-6 and ammonia in blood were done. Diagnosis of MHE is based on psychometric hepatic encephalopathy score (PHES) which includes digit symbol test (DST), number connection test-A (NCT-A), number connection test-B (NCT-B), serial dotting test (SDT), and line drawing test (LDT).

Results: The mean age was $52.0 \pm 7.47$ in MHE patients. Most of the MHE patients were males. Most of the patients with MHE were working other jobs and were not drivers (63.2\% vs 36.8\%). MHE was observed in 19 patients (25.7\%). Albumin, interleukin-6 (IL-6), and ammonia were statistically significantly different between both groups.

Conclusion: Minimal hepatic encephalopathy (MHE) was observed in $25.7 \%$ of patients which points that we must give attention in the screening of MHE as road traffic accident is considered the main cause of mortality in our community with high incidence of liver disease.
\end{abstract}

Keywords: Minimal hepatic encephalopathy, Road traffic accidents, Egypt

\section{Introduction}

Hepatic encephalopathy (HE) is a manifestation of neuropsychiatric symptoms (after exclusion of another brain disease) in patients with liver cirrhosis. It is characterized by personality changes, cognitive dysfunction, and altered level of consciousness [1].

The term minimal hepatic encephalopathy (MHE) is the encephalopathy that does not associate with clinically overt cognitive dysfunction but can be diagnosed by neuropsychological tests [2]. Patients with MHE have no known clinical criteria of HE but have only mild psychomotor and cognitive impairment. Minimal hepatic

\footnotetext{
* Correspondence: Mohnegm2@yahoo.com

Deceased

${ }^{3}$ Faculty of Medicine, Suez Canal University, Ismailia, Egypt

Full list of author information is available at the end of the article
}

encephalopathy (MHE) may have a bad impact on quality of life, risk of road traffic accidents, and difficulties in daily life and can be converted to overt HE [3, 4].

Navigation is a collection of processes needed for driving safely and requires that working memory, attention, and speed of mental processing are intact. Navigation difficulty can be found in patients with cirrhosis and MHE which is characterized by poor attention and response inhibition [5].

Minimal hepatic encephalopathy (MHE) can be coupled with poor driving skills and a higher risk of motor car accidents due to poor attention and lack of visuomotor coordination $[6,7]$. Poor psychometric performance is associated with driving difficulties [8].

Standard neurological examination is not enough to diagnose subtle cognitive function deficit, so the psychometric 
methods are widely used tests in the evaluation of patients with MHE $[2,9,10]$.

\section{Aim}

The aim of this study is to identify the incidence of minimal hepatic encephalopathy in patients actively involved in a road traffic accident and its role in the increased incidence of road traffic accidents.

\section{Patients and methods}

The study is a cross-sectional comparative study for evaluation and screening of minimal hepatic encephalopathy in road traffic accident victims attending the emergency departments in the Suez Canal University Hospital. Seventy-four consecutive patients were included in the study according to the inclusion and exclusion criteria.

We included all patients involved in road traffic accidents (drivers, history of fall, pedestrians whom accidents occur during crossing the road); patients with clinically proven hepatic cirrhosis, without clinical manifestation of HE; and patients with age range between 18 and 65 years.

We excluded patient with grade1, 2, 3, or $4 \mathrm{HE}$; patients presented with head trauma, fracture of the dominant hand used in writing, and recent GIT bleeding (within the previous 4 weeks); and patients with renal disease (serum creatinine $>1.5 \mathrm{mg} / \mathrm{dl}$ ), respiratory disease, congestive heart failure, diabetes, hypertension, and severe malnutrition. Patients with neurological disease and psychiatric problems (e.g., Alzheimer's disease, Parkinson's disease, depression) which may affect mental functions or ophthalmological disorders were also excluded. No patients had been prescribed lactulose or neomycin in the previous 6 weeks, and none was receiving antibiotic or sedatives in the week prior to the study. Patients with psychoactive drugs, anti-depressants, or sedatives in the last 6 weeks were excluded. Patients with a history of alcohol intake were excluded. Patients with hepatocellular carcinoma, transjugular intrahepatic portosystemic shunt (TIPS), or shunt surgery were excluded. Patients receiving antiviral treatment before or during the study were excluded. Patients with serum sodium $<130 \mathrm{mmol} / \mathrm{L}$ and serum potassium $<3.2 \mathrm{mmol} / \mathrm{L}$ or $>5 \mathrm{mmol} / \mathrm{L}$ were excluded. Pregnant females and illiterate subjects ere excluded.

Thorough history taking and neurological examinations were done for the exclusion of patients with neurological disease and psychiatric problems (e.g., Parkinson's disease, depression). Mental state examination using standardized mini-mental state examination (SMMSE) was done for the exclusion of patients with Alzheimer's disease.

Laboratory investigations include routine laboratory investigations (complete blood count $(\mathrm{CBC})$, liver function tests, hepatitis markers, kidney function tests, random blood sugar, serum sodium, serum potassium, and serum calcium) and determination of interleukin-6 (IL-6) in serum and ammonia in blood.

Imaging done in the ER includes pelvi-abdominal ultrasound for the exclusion of patients with hepatocellular carcinoma and pregnant females and brain CT for the exclusion of patients with traumatic brain injury.

\section{Psychometric hepatic encephalopathy score}

Psychometric hepatic encephalopathy score (PHES) was the base for diagnosis of MHE; it includes "digit symbol test (DST), number connection test-A (NCT-A), number connection test-B (NCT-B), serial dotting test (SDT), and line drawing test (LDT)." A score is defined as "the number of standard deviations of the difference between the two values for each test"; MHE was diagnosed with the sum of all scores less than or equal to -4 points [11]. According to the guidelines of the International Society for Hepatic Encephalopathy and Nitrogen Metabolism [2], "the results of NCT-A will be considered abnormal when the test scores are more than the mean +2 standard deviations (SD) from the age-matched normal values, and DST will be considered abnormal when the test scores are less than the mean - 2 SD from the age-matched normal values."

\section{The number connection test}

The NCT-A is the "testing of visuo-spatial orientation and psychomotor speed." The patient is given a paper with 25 numbered circles randomly spread on the paper. The patient is asked to connect the circles from 1 to 25 as quick as possible. The test result is "the time needed by the subject including error correction time" [2]. The NCT-B is nearly like the NCT-A. The numbers from 1 to 13 and the letters from $\mathrm{A}$ to $\mathrm{L}$ were included in the circles. "The patients are asked to connect the numbers and letters in an alternating manner, that means go from 1-A-2-B-3-C and so on." The test result represents the time needed including error correction time [2].

\section{The digit symbol test}

The patients take a series of double boxes with a number given in the upper part. "The task is to draw a symbol pertinent to this number into the lower part of the boxes. Nine fixed pairs of numbers and symbols are given at the top of the test sheet." The number of boxes correctly filled within $90 \mathrm{~s}$ is the test result [2].

\section{The line drawing test}

The patients are asked to track a route in a labyrinth without borderline crossing or touching. The route is divided into small sections, and when the patient touches or crosses the border in a certain section, this is counted. The test results include mistake number and the time required to go through the labyrinth [2]. 


\section{The circle dotting test}

This test is the testing of pure motor speed and considered the simplest test of the battery. The patient is given a sheet composed of 100 circles, and the patient is asked to put a dot in each circle; the patient is prepared by dotting the 20 circles at the top of the sheet, and the time needed is the test result [11].

\section{Statistical analysis}

IBM SPSS statistics (V. 21.0, IBM Corp., USA, 2012) was used for data analysis. Quantitative data was expressed as median or means \pm standard deviation $(\mathrm{SD})$ as appropriate. Qualitative data was expressed as frequency (numbers) and percentages. The results of all categorical variables were given in the form of rates (\%). Student $t$ test will be used to test the significance of difference for quantitative variables that follow a normal distribution. Chi-squares and Fisher's exact tests were used to test the significance of difference for qualitative variables.

The independent data of the study was conducted and analyzed. Definitive statistics will be used for the analysis of the sociodemographic and other variables. Firstly, the relation between the dependent and independent variables was studied using the chi-square test and the $t$ test. Second, the significant variables were subjected to multivariate logistic regression analysis.

\section{Ethical considerations}

Data had been collected without any harm to the patients and after explaining the aim of the study to the patients, and an informed written consent was obtained; they did are not exposed to any harm or additional invasiveness.

The study was approved by the ethical committee of Faculty of Medicine, Suez Canal University on 18 June 2013 (Research\# 996).

\section{Results}

The study was conducted on 74 patients, age range between 20 and 62 (mean 40.59); 93.2\% of them were male, only $6.8 \%$ were female. Driver represents $32.4 \%$ of the patients, rural residency represents $56.8 \%$, and married patients represent $74.3 \%$. Most of the patients (68.9) are drivers for more than 10 years, $82.4 \%$ of the patients have no traffic mistakes in the last year, $73.0 \%$ has not following the traffic rules, and $82.4 \%$ has no previous history of traffic accidents.

We found that $19(25.7 \%)$ of the patients have abnormal psychometric hepatic encephalopathy score and consequently diagnosed as having MHE.

The mean of total bilirubin was found to be 1.20 , direct bilirubin 0.66 , albumin 3.79, prothrombin time (PT) 13.40, alanine aminotransferase (ALT) 31.01, aspartate aminotransferase (AST) 31.90, creatinine 0.85 , sodium (Na) 138.05, potassium (K) 4.13, calcium (Ca) 9.00, hemoglobin $(\mathrm{Hb}) 12.89$, total leucocyte count (TLC)
9.295, platelet 251.62, ammonia 75.68, and interleukin-6 (IL-6) 168.02, and the mental state examination using standardized mini mental state examination (SMMSE) score was 27.43 . Only 16 patients found to have hepatitis $\mathrm{C}$ virus (HCV) $\mathrm{Ab}(21.6 \%)$, and 1 patient have hepatitis $B$ virus surface antigen (HBsAg) (1.4\%).

There was a statistically significant difference between normal and abnormal psychometric hepatic encephalopathy scores regarding the age and marital status. However, there were no statistically significant differences between both groups regarding the other descriptive data (Table 1).

Among patients who have abnormal psychometric hepatic encephalopathy score (19), $89.5 \%$ of them reported no traffic mistakes in the last year, $57.9 \%$ are following the traffic rules, and $84.2 \%$ have previous history of traffic accidents.

There was a statistically significant difference between normal and abnormal psychometric hepatic encephalopathy score regarding the total bilirubin, albumin, AST, creatinine, $\mathrm{Na}$, Ca, ammonia, IL-6, and HCV Ab (Table 2).

We found that most patients that are not following the traffic rules are young, males, drivers, rural resident, and married (Table 3).

None of the patients that follow the traffic rules reported traffic mistakes in the last year or report a history of traffic accidents while $24.1 \%$ of the patients that do not follow traffic rules reported traffic mistakes in the last year and a history of traffic accidents.

According to laboratory parameters, we found that patients that follow the traffic rules have lower ammonia

Table 1 Psychometric hepatic encephalopathy score and descriptive data

\begin{tabular}{|c|c|c|c|c|}
\hline & \multicolumn{2}{|c|}{$\begin{array}{l}\text { Psychometric hepatic encephalopathy } \\
\text { score }\end{array}$} & \multirow[t]{3}{*}{$t$ test } & \multirow[t]{3}{*}{$P$ value } \\
\hline & Abnormal (19) & Normal (55) & & \\
\hline & Mean $\pm S D$ & & & \\
\hline \multirow[t]{2}{*}{ Age } & $52.0 \pm 7.47$ & $36.65 \pm 11.16$ & 5.563 & $<0.001^{* *}$ \\
\hline & No (\%) & & $x^{2}$ & $P$ value \\
\hline \multicolumn{5}{|l|}{ Sex } \\
\hline Male & 19 (100) & $50(90.9)$ & 1.852 & 0.174 \\
\hline Female & 0 & $5(9.1)$ & & \\
\hline \multicolumn{5}{|l|}{ Occupation } \\
\hline Driver & $7(36.8)$ & $17(30.9)$ & 0.227 & 0.634 \\
\hline Other jobs & $12(63.2)$ & $38(69.1)$ & & \\
\hline \multicolumn{5}{|l|}{ Residence } \\
\hline Urban & $8(42.1)$ & 24 (43.6) & 0.013 & 0.908 \\
\hline Rural & $11(57.9)$ & $31(56.4)$ & & \\
\hline \multicolumn{5}{|l|}{ Marital status } \\
\hline Married & $19(100)$ & $36(65.5)$ & 8.831 & $0.003^{* *}$ \\
\hline Single & 0 & $19(34.5)$ & & \\
\hline
\end{tabular}

$X^{2}$ chi-square test. ${ }^{*} P<0.005$ is highly significant, $P>0.05$ is not significant 
Table 2 Psychometric hepatic encephalopathy score and lab data

\begin{tabular}{|c|c|c|c|c|}
\hline & \multicolumn{2}{|c|}{ Psychometric hepatic encephalopathy score } & \multirow[t]{3}{*}{$t$ test } & \multirow[t]{3}{*}{$P$ value } \\
\hline & Abnormal (19) & Normal (55) & & \\
\hline & \multicolumn{2}{|l|}{ Mean \pm SD } & & \\
\hline \multicolumn{5}{|l|}{ Lab investigation } \\
\hline Total bilirubin & $2.54 \pm 5.26$ & $0.74 \pm 0.26$ & 2.572 & $0.012^{*}$ \\
\hline Albumin & $3.23 \pm .62$ & $3.99 \pm 0.41$ & 5.985 & $<0.001^{* *}$ \\
\hline $\mathrm{PT}^{1}$ & $13.98 \pm 2.61$ & $13.19 \pm 1.43$ & 1.651 & 0.103 \\
\hline $\mathrm{ALT}^{2}$ & $36.63 \pm 23.31$ & $29.07 \pm 15.66$ & 1.588 & 0.117 \\
\hline $\mathrm{AST}^{3}$ & $46.42 \pm 45.77$ & $26.89 \pm 11.22$ & 2.951 & $0.004^{* *}$ \\
\hline Creatinine & $1.20 \pm 1.07$ & $0.72 \pm 0.17$ & 3.166 & $0.002^{* *}$ \\
\hline $\mathrm{Na}^{4}$ & $135.4 \pm 4.64$ & $138.9 \pm 3.9$ & 3.173 & $0.002^{* *}$ \\
\hline$K^{5}$ & $4.0 \pm 0.52$ & $4.18 \pm 0.38$ & 1.599 & 0.114 \\
\hline $\mathrm{Ca}^{6}$ & $8.46 \pm 0.75$ & $9.18 \pm 0.29$ & 5.948 & $<0.001^{* *}$ \\
\hline $\mathrm{Hb}^{7}$ & $12.57 \pm 2.28$ & $13.0 \pm 1.72$ & 0.845 & 0.401 \\
\hline$T_{L C}^{8}$ & $9.90 \pm 4.02$ & $9.08 \pm 3.73$ & 0.810 & 0.421 \\
\hline $\mathrm{PLT}^{9}$ & $237.1 \pm 113.1$ & $256.6 \pm 123.2$ & 0.851 & 0.398 \\
\hline Ammonia & $66.47 \pm 13.68$ & $78.87 \pm 21.5$ & 2.898 & $0.006^{* *}$ \\
\hline \multirow[t]{2}{*}{ IL-6 $6^{10}$} & $215.4 \pm 38.4$ & $151.6 \pm 34.1$ & 6.815 & $<0.001^{* *}$ \\
\hline & \multicolumn{2}{|l|}{ No (\%) } & $x^{2}$ & $P$ value \\
\hline \multicolumn{5}{|l|}{$\mathrm{HbsAg}^{11}$} \\
\hline Positive & $1(5.3)$ & 0 & 2.934 & 0.087 \\
\hline Negative & $18(94.7)$ & $55(100)$ & & \\
\hline \multicolumn{5}{|l|}{$\mathrm{HCV} A b^{12}$} \\
\hline Positive & $16(84.2)$ & 0 & 59.093 & $<0.001^{*}$ \\
\hline Negative & $3(15.8)$ & $55(100)$ & & \\
\hline
\end{tabular}

$X^{2}$ chi-square test. ${ }^{*} P<0.05$ is significant, ${ }^{* *} P<0.005$ is highly significant, and $P>0.05$ is not significant

${ }^{1}$ Prothrombin time

${ }^{2}$ Alanine aminotransferase

${ }^{3}$ Aspartate aminotransferase

${ }^{4}$ Sodium

${ }^{5}$ Potassium

${ }^{6}$ Calcium

${ }^{7}$ Hemoglobin

${ }^{8}$ Total leucocyte count

${ }^{9}$ platelet

${ }^{10}$ Interleukin-6

${ }^{11}$ hepatitis $B$ virus surface antigen

${ }^{12}$ hepatitis $C$ virus $A b$

level $(68.4 \pm 15.63)$ compared with patients that do not following the traffic rules $(78.38 \pm 21.51)$ with statistically significant differences between both groups. Also, we found that patients who had traffic mistakes in the last year had higher ammonia level $(81.923 \pm 19.86)$ compared with patients that had no traffic mistakes $(74.36 \pm 20.52)$ with no statistically significant differences between both groups.

There were no statistically significant differences between both groups whether following and not following the traffic rules regarding the hepatitis markers. Ten out of 17 patients that have positive hepatitis markers are not following the traffic rules. Also, 11 out of 19 patients that have abnormal psychometric hepatic encephalopathy score are not following the traffic rules with no statistically significant differences between both groups. Only 2 patients (14.4\%) with abnormal psychometric hepatic encephalopathy score reported to have traffic mistakes in the last year. We found that all patients that are not following the traffic rules do traffic mistakes, and $53.8 \%$ has a history of traffic accident.

There was a statistically significant difference between both groups that had and had no traffic mistakes in the last year regarding the occupation and residence. However, there were no statistically significant 
Table 3 Descriptive data and degree of following the traffic rules

\begin{tabular}{|c|c|c|c|c|}
\hline & \multicolumn{2}{|c|}{ Following the traffic rules } & \multirow[t]{2}{*}{$t$ test } & \multirow[t]{2}{*}{$P$ value } \\
\hline & Yes (20) & No (54) & & \\
\hline & Mean \pm SD & & & \\
\hline \multirow[t]{2}{*}{ Age } & $44.05 \pm 12.04$ & $39.31 \pm 12.26$ & 1.482 & 0.143 \\
\hline & No (\%) & & $x^{2}$ & $P$ value \\
\hline \multicolumn{5}{|l|}{ Sex } \\
\hline Male & $18(90)$ & $51(94.4)$ & 0.458 & 0.499 \\
\hline Female & $2(10)$ & $3(5.6)$ & & \\
\hline \multicolumn{5}{|l|}{ Occupation } \\
\hline Driver & $6(30)$ & $18(33.3)$ & 0.074 & 0.786 \\
\hline Other jobs & $14(70)$ & $36(66.7)$ & & \\
\hline \multicolumn{5}{|l|}{ Residence } \\
\hline Urban & $8(40)$ & $24(44.4)$ & 0.117 & 0.732 \\
\hline Rural & $12(60)$ & $30(55.6)$ & & \\
\hline \multicolumn{5}{|l|}{ Marital status } \\
\hline Married & $17(85)$ & $38(70.4)$ & 1.637 & 0.201 \\
\hline Single & $3(15)$ & $16(29.6)$ & & \\
\hline
\end{tabular}

differences between both groups regarding the age, sex, and marital status (Table 4).

The statistical significant predicted risk factors for the development of MHE were age and smoking where with increasing age, the odd ratio and hazard increase 1.572

Table 4 Descriptive data according to the traffic mistakes in the last year

\begin{tabular}{|c|c|c|c|c|}
\hline & \multicolumn{2}{|c|}{ Traffic mistakes } & \multirow[t]{3}{*}{$t$ test } & \multirow[t]{3}{*}{$P$ value } \\
\hline & Yes (13) & No (61) & & \\
\hline & \multicolumn{2}{|l|}{ Mean \pm SD } & & \\
\hline \multirow[t]{2}{*}{ Age } & $38.23 \pm 9.63$ & $41.09 \pm 12.81$ & 0.760 & 0.450 \\
\hline & \multicolumn{2}{|l|}{ No (\%) } & $x^{2}$ & $P$ value \\
\hline \multicolumn{5}{|l|}{ Sex } \\
\hline Male & $12(92.3)$ & $57(93.4)$ & 0.022 & 0.882 \\
\hline Female & $1(7.7)$ & $4(6.6)$ & & \\
\hline \multicolumn{5}{|l|}{ Occupation } \\
\hline Driver & $8(61.5)$ & $16(26.2)$ & 6.097 & $0.014^{*}$ \\
\hline Other jobs & $5(38.5)$ & $45(73.8)$ & & \\
\hline \multicolumn{5}{|l|}{ Residence } \\
\hline Urban & $9(69.2)$ & $23(37.7)$ & 4.339 & $0.037^{*}$ \\
\hline Rural & $4(30.8)$ & $38(62.3)$ & & \\
\hline \multicolumn{5}{|l|}{ Marital status } \\
\hline Married & $8(61.5)$ & $47(77)$ & 1.351 & 0.245 \\
\hline Single & $5(38.5)$ & $14(23)$ & & \\
\hline
\end{tabular}

$X^{2}$ chi-square test. ${ }^{*} P<0.05$ is significant, $P>0.05$ is not significant in older age than in younger age, and in smoking, the odd ratio and hazard increase 8.070 than in non-smoking (Table 5).

\section{Discussion}

In patients with cirrhosis, minimal hepatic encephalopathy (MHE) is a diagnosis that must be considered and is associated with a poor quality of life [12], fall risk increased [13], and driving a motor vehicle becomes difficult [14]. So, a concern that the term "minimal" may underestimate the condition raise the assumption that this disease stage may be renamed covert encephalopathy [15].

In our study, there was a statistically significant difference between normal and abnormal psychometric hepatic encephalopathy scores regarding the age and marital status. However, there were no statistically significant differences between both groups regarding the other descriptive data. The age range was between 20 and 62 years where $93.2 \%$ of them were male. This is in agreement with other studies [16-19] who stated that age can influence neuropsychological performance in patients with MHE. Other studies showed that there was no effect of age or gender on the diagnosis of MHE $[7,20]$.

In our study, we noticed that the level of ammonia was significantly higher in patients with MHE. These results were in agreement with Gad et al. [21]. However, these results were not in agreement with $\mathrm{Li}$ et al. [18] who reported that MHE did not correlate with venous ammonia levels which can be explained that in MHE patients, the blood-brain barrier may be breached, enabling ammonia to diffuse across the blood-brain barrier into the brain more freely, as such, the venous ammonia concentration of patients with MHE may be similar to patients without MHE [22].

Table 5 Predicted risk factors for the development of MHE by multivariate logistic regression analysis

\begin{tabular}{lllll}
\hline & $P$ value & Odds ratio & \multicolumn{2}{c}{$95 \% \mathrm{Cl}$} \\
\cline { 5 - 6 } & & & Lower & Upper \\
\hline Age & $0.012^{*}$ & 1.572 & 1.036 & 1.325 \\
Occupation & 0.256 & 0.367 & 0.065 & 2.071 \\
Residence & 0.959 & 0.954 & 0.161 & 5.646 \\
Smoking & $0.050^{*}$ & 8.070 & 0.969 & 67.227 \\
Drugs & 0.834 & 1.199 & 0.220 & 6.542 \\
Driving duration & 0.194 & 0.066 & 0.001 & 3.970 \\
Duration of driving license & 0.103 & 18.934 & 0.553 & 648.603 \\
Traffic mistakes in last year & 0.902 & 0.846 & 0.059 & 12.181 \\
Degree of following the & 0.374 & 0.433 & 0.068 & 2.741 \\
traffic rules & & & & \\
Previous history of traffic & 0.787 & 1.358 & 0.147 & 12.542 \\
accidents & & & & \\
\hline Cl confidence interval & & & &
\end{tabular}


In our study, 19 patients (25.7\%) were found to have MHE according to the assessment by psychometric hepatic encephalopathy score. This finding is consistent with Cordoba [23] who reported MHE in $28.6 \%$. Also, Seo et al. [19] reported that minimal hepatic encephalopathy was detected in $25.6 \%$. Bass et al. [24] reported that the percentage of MHE in cirrhotic patients is from 20 to $85 \%$. Amodio et al. [25] reported that the percentage is about 15\%, and Quero et al. [26] reported that up to $27 \%$ of cirrhotic patients have MHE. On the other hand, Sood et al. [27] reported that up to $75 \%$ of cirrhotic patients have MHE, and Dhiman et al. [28] reported that MHE was detected in $48 \%$. Also, our results were not consistent with a study conducted at the Hepatology Outpatient Clinic of Mansoura Specialized Medical Hospital, Egypt, which found that 47\% showed evidence for MHE [21]. This difference in the percentage of patients with MHE is due to some research considers grade 0 and 1 ; however, we consider only grade 0 according to West Haven classification system, criteria of patient selection, difference in diagnostic criteria [29], and the variability of tests used [30-32].

We noticed that $17.6 \%$ of patients had traffic mistakes in the last year and $73 \%$ of patients not following the traffic rules, and consequently, there is an increase in the amount of paid traffic fines in the last year, and only $17.6 \%$ of patients had a previous history of traffic accidents. They noted a decrease in their attention during driving or other manual works. Some of them reported an increase in the speed of their driving, others reported illegal turns and other mistakes during crossing intersections, some of them reported delayed response to the flickering lights of other cars, and others reported decreasing ability on driving during the night. However, the results of our study reported that there were no significant differences between both groups regarding the significance of psychometric hepatic encephalopathy score. These results come in agreement with the results and recommendations of the Egyptian Annual Conference of Hepatology in February 2010 [33] which reported that there was an increase in the risk of motor violations and accidents (MVAs) in patients with hepatic diseases especially patients with MHE and the rate of accidents may reach up to $20 \%$ of the total accidents.

Wein et al. [6] and Bajaj et al. [7] reported similar results and showed that there is a great link between MHE and the traffic violations or motor vehicle accidents. Rizzo et al. [34] reported that a decline in cognitive functions increases the risk of automobile crashes, due to the impairment of attention and speed of mental processing. Both of which are characteristics of MHE, which affect the individual ability to react to unexpected traffic conditions such as illegal incursions by another vehicle at an intersection.
Lastly, in our study, we reported a multivariate analysis for different risk factors for the development of MHE as the predicted risk factors for the development of MHE were age and smoking where with increasing age, the odd ratio and hazard increase 1.572 in older age than in younger age, and in smoking, the odd ratio and hazard increase 8.070 in than non-smoking. This comes in agreement with Gad et al. [21] who reported smoking as a significant risk factor for MHE. These results were not the same as Seo et al. [19] who reported that education years, Child-Pugh score (consists of five clinical features: total bilirubin, serum albumin, prothrombin time, ascites, and hepatic encephalopathy and is used to assess the prognosis of chronic liver disease and cirrhosis), and venous ammonia level were independently associated with MHE on multivariate analysis.

In conclusion, standard mental and neurological examinations may fail to detect mild changes in the central nervous system functions in patients with compensated liver cirrhosis. Neuro-psychological tests can detect these abnormalities. Minimal hepatic encephalopathy (MHE) is common among cirrhotic patients and consequently can impair safe driving and judgment during driving so incidence of traffic violations and accidents increased in those patients.

The main limitation of our study was the lack of other diagnostic methods for minimal hepatic encephalopathy other than psychometric hepatic encephalopathy score (PHES). Also, the accuracy of performance of PHES may be related mainly to the level of education which means that the best results will be obtained if the test will be done at higher educational level. Although neurophysiological tests as electroencephalography (EEG) have considerable advantages, their use is limited by the availability of apparatus in the emergency room, need time for acquiring the technic and analyzing the results.

\section{Abbreviations \\ DST: Digit symbol test; HE: Hepatic encephalopathy; LDT: Line drawing test: MHE: Minimal hepatic encephalopathy; NCT-A: Number connection test-A; NCT-B: Number connection test -B; PHES: Psychometric hepatic \\ encephalopathy score; SDT: Serial dotting test; TIPS: Transjugular intrahepatic portosystemic shunt}

\section{Acknowledgements}

Not applicable

\section{Funding}

Not applicable

\section{Availability of data and materials}

The data that support the findings of this study are available from Faculty of Medicine, Suez Canal University, Egypt but restrictions apply to the availability of these data, which were used under license for the current study, and so are not publicly available. Data are however available from the authors upon reasonable request and with permission of Faculty of Medicine, Suez Canal University, Egypt. 


\section{Authors' contributions}

AME participated in the idea and design of the study. EAE participated in the design of the study, guidance, follow-ups, and final revision. AF led the practical work and follow-ups and guided the laboratory work and final revision. MN participated in the design of the study, prepared the paper, and participated in the sequence alignment and drafting of the manuscript and its final revision. SM contributed to the practical work, participated in the design of the study, and performed the statistical analysis. OW contributed to the practical work and participated in the sequence alignment and drafting of the manuscript. All authors read and approved the final manuscript.

\section{Ethics approval and consent to participate}

An informed written consent was taken from each patient. All data obtained from every patient were confidential and were not used outside the study. The patients have rights to withdraw from the study at any time without giving any reason. All the cost of the investigations was afforded by the researcher. The study was approved from ethical committee Faculty of Medicine, Suez Canal University on 18/6/2013 (Research\# 996).

\section{Consent for publication}

Not applicable

\section{Competing interests}

The authors declare that they have no competing interests.

\section{Publisher's Note}

Springer Nature remains neutral with regard to jurisdictional claims in published maps and institutional affiliations.

\section{Author details}

${ }^{1}$ Suez Canal University, Ismailia, Egypt. ${ }^{2}$ Suez Canal University, Ismailia, Egypt. ${ }^{3}$ Faculty of Medicine, Suez Canal University, Ismailia, Egypt. ${ }^{4}$ Suez Canal University, Ismailia, Egypt.

Received: 6 December 2017 Accepted: 4 January 2019

Published online: 18 January 2019

\section{References}

1. David C Wolf. Encephalopathy, Hepatic, eMedicine Specialties, Gastroenterology, Liver, Updated: Mar 25. 2010.

2. Randolph C, Hilsabeck R, Kato A, Kharbanda P, Li YY, Mapelli D, Weissenborn K. Neuropsychological assessment of hepatic encephalopathy: ISHEN practice guidelines. Liver Int. 2009;29(5):629-35.

3. Dhiman RK, Chawla YK. Minimal hepatic encephalopathy. Indian J Gastroenterol. 2009;28(1):5-16.

4. Bajaj JS, Heuman DM, Bell DE, White M, Gilles H, Luketic VA, Sanyal AJ. Burden of cirrhosis and hepatic encephalopathy on patients and caregivers. Hepatology. 2010;52:410A.

5. Romero-Gómez M, Boza F, García-Valdecasas MS, García E, Aguilar-Reina J. Subclinical hepatic encephalopathy predicts the development of overt hepatic encephalopathy. Am J Gastroenterol. 2001;96(9):2718-23.

6. Wein C, Koch H, Popp B, Oehler G, Schauder P. Minimal hepatic encephalopathy impairs fitness to drive. Hepatology. 2004;39(3):739-45.

7. Bajaj JS, Hafeezullah M, Hoffmann RG, Saeian K. Minimal hepatic encephalopathy: a vehicle for accidents and traffic violations. Am J Gastroenterol. 2007;102(9):1903-9.

8. Bajaj JS, Hafeezullah M, Hoffmann RG, Varma RR, Franco J, Binion DG, Saeian K. Navigation skill impairment: another dimension of the driving difficulties in minimal hepatic encephalopathy. Hepatology. 2008;47(2):596-604.

9. Weissenborn K, Ennen JC, Schomerus H, Ruckert N, Hecker H. Neuropsychological characterization of hepatic encephalopathy. Journal of Hepatology. 2001;34:768-73.

10. Qi R, Zhang L, Wu S, Zhong J, Zhang Z, Zhong Y, Wu X. Altered restingstate brain activity at functional MR imaging during the progression of hepatic encephalopathy. Radiology. 2012;264(1):187-95.

11. Montoliu C, Piedrafita B, Serra MA, Del Olmo JA, Urios A, Rodrigo JM, Felipo V. IL-6 and IL-18 in blood may discriminate cirrhotic patients with and without minimal hepatic encephalopathy. J Clin Gastroenterol. 2009;43(3): 272-9.
12. Groeneweg M, Quero JC, De Bruijn I, Hartmann IJ, Essink-bot ML, Hop WC Schalm SW. Subclinical hepatic encephalopathy impairs daily functioning. Hepatology. 1998;28(1):45-9.

13. Román E, Córdoba J, Torrens M, Torras X, Villanueva C, Vargas V, Soriano G. Minimal hepatic encephalopathy is associated with falls. Am J Gastroenterol. 2011;106(3):476-82.

14. Kircheis G, Knoche A, Hilger N, Manhart F, Schnitzler A, Schulze H, Häussinger D. Hepatic encephalopathy and fitness to drive. Gastroenterology. 2009;137(5):1706-15.

15. Kappus MR, Bajaj JS. Assessment of minimal hepatic encephalopathy (with emphasis on computerized psychometric tests). Clinics in liver disease. 2012 16(1):43-55

16. Zeneroli ML, Cioni G, Ventura P, Russo AM, Venturini I, Casalgrandi G, Ventura E. Interindividual variability of the number connection test. J Hepatol. 1992;15(1-2):263-4.

17. Lezak MD, Howieson DB,Loring DW. Neuropsychological Assessment. 4thed. New York, NY: Oxford University Press. 2004

18. Li SW, Wang K, Yu YQ, Wang HB, Li YH, Xu JM. Psychometric hepatic encephalopathies score for diagnosis of minimal hepatic encephalopathy in China. World J Gastroenterol. 2013;19(46):8745-51.

19. Seo YS, Yim SY, Jung JY, Kim CH, Kim JD, Keum B, Ryu HS. Psychometric hepatic encephalopathies score for the detection of minimal hepatic encephalopathy in Korean patients with liver cirrhosis. J Gastroenterol Hepatol. 2012;27(11):1695-704.

20. Bajaj JS, Wade JB, Sanyal AJ. Spectrum of neurocognitive impairment in cirrhosis: implications for the assessment of hepatic encephalopathy. Hepatology. 2009;50(6):2014-21.

21. Gad YZ, Zaher AA, Moussa NH, El-desoky AEE, Al-Adarosy HA. Screening for minimal hepatic encephalopathy in asymptomatic drivers with liver cirrhosis. Arab Journal of Gastroenterology. 2011;12(2):58-61.

22. McPhail MJ, Bajaj JS, Thomas HC, Taylor-Robinson SD. Pathogenesis and diagnosis of hepatic encephalopathy. Expert Rev Gastroenterol Hepatol. 2010;4(3):365-78.

23. Córdoba J. New assessment of hepatic encephalopathy. J Hepatol. 2011 54(5):1030-40

24. Bass N, Leevy C, Munoz S, et al. Advancing the diagnosis and management of hepatic encephalopathy. Gastoenterol Hepatol. 2007;3(suppl 5):739-45.

25. Amodio P, Marchetti P, Del Piccolo F, Rizzo C, lemmolo RM, Caregaro L, Gatta A. Study on the Sternberg paradigm in cirrhotic patients without overt hepatic encephalopathy. Metab Brain Dis. 1998;13(2):159-72.

26. Quero JC, Hartmann IJ, Meulstee J, Hop WC, Schalm SW. The diagnosis of subclinical hepatic encephalopathy in patients with cirrhosis using neuropsychological tests and automated electroencephalogram analysis. Hepatology. 1996;24(3):556-60.

27. Sood GK, Sarin SK, Mahaptra J, Broor SL. Comparative efficacy of psychometric tests in detection of subclinical hepatic encephalopathy in nonalcoholic cirrhotics: search for a rational approach. Am J Gastroenterol. 1989;84(2):156-9.

28. Dhiman RK, Saraswat VA, Sharma BK, Sarin SK, Chawla YK, Butterworth R, Madan K. Minimal hepatic encephalopathy: consensus statement of a working party of the Indian National Association for Study of the Liver. J Gastroenterol Hepatol. 2010;25(6):1029-41.

29. Koch H, Schauder P, Schäfer G, Dahme B, Ebel W, Vahldiek B, Henning H. Diagnosis and prevalence of latent hepatic encephalopathy. Zeitschrift fur Gastroenterologie. 1990;28(11):610-5.

30. Moore JW, Dunk AA, Crawford JR, Deans H, Besson JAO, De Lacey G, et al. Neuropsychological deficits and morphological MRI brain scan abnormalities in apparently healthy non-encephalopathic patients with cirrhosis: a controlled study. J Hepatol. 1989;9(3):319-25.

31. Ferenci $P$, Lockwood A, Mullen K, Tarter R, Weissenborn K, Blei AT. Hepatic encephalopathy_definition, nomenclature, diagnosis, and quantification: final report of the working party at the 11th World Congresses of Gastroenterology, Vienna, 1998. Hepatology. 2002;35(3):716-21.

32. Ortiz M, Jacas C, Córdoba J. Minimal hepatic encephalopathy: diagnosis, clinical significance and recommendations. J Hepatol. 2005:42(1):S45-53.

33. Egyptian Annual Conference Of Hepatology. "Results and recommendations" available at Egyptian Society of Hepatology. 2010 website www.egyptgastrohep.com/email/

34. Rizzo M, McGehee DV, Dawson JD, Anderson SN. Simulated car crashes at intersections in drivers with Alzheimer disease. Alzheimer Dis Assoc Disord. 2001;15(1):10-20 